\title{
Molecular Dynamic Investigations of the Behavior of Trinidad \& Tobago Asphaltenes in Process fluids - Validation of the GROMACS software.
}

\author{
Festus Addo-Yobo ${ }^{1}$, Adrian Lutchman ${ }^{2}$
}

\begin{abstract}
Several nations in the Caribbean sub-region have abundant resources of heavy oils which contain asphaltenes. These oils can be mined and monetized for national development, however successful processing of this resource is impeded by the unpredictable behavior of the asphaltenes such as precipitation, which can lead to pipe blockage during transport, and fouling of process equipment, thus adding to costs.
\end{abstract}

In this study we use molecular dynamic techniques to simulate asphaltene-asphaltene and asphaltene-solvent interactions and investigate how these might be affected by temperature. The motion of the molecules is determined by the application of Newton's laws in potential field described by the Optimized Potential for Liquid Simulation All Atoms (OPLS_AA). The Particle-mesh Ewald (PME) algorithm is used to calculate electrostatic interactions. The periodic boundary condition (PBC) and the Maxwell-Boltzmann distribution of the initial velocities are adopted. We use the Nose-Hoover thermostat and the Parrinello-Rahman barostat to control temperature and pressure in constant volume, constant temperature (NVT) simulations.

The results of atomistic MD simulations can be presented by videographics and $\mathrm{U}$-Tube, and also by Cartesian graphical relationships between cluster size and time. The effect of temperature on the clustering process is demonstrated within the constraints of the simulations: 24 ns, 50 asphaltene molecules in $14 \%$ wt/wt composition.

Keywords-Molecular dynamics, asphaltenes, GROMACS, heavy oils

\section{Introduction}

Trinidad and Tobago heavy oils contain asphaltenes which can precipitate unpredictably and cause disruptions in transportation and processing such as coking, well and pipe blockage and unwanted stabilization of oil-water emulsion1. The mechanisms underlying the above are not understood and therefore cannot be taken into account at the design stage to save costs. Asphaltenes comprise many species of molecules with high carbon to hydrogen ratios, aromatic cores, heteroatoms and aliphatic chains (see Fig. 1 below).

The research in this LMSEE laboratory though in its infancy, aims to use Molecular Dynamics (MD) to provide insight into the behaviour of asphaltenes contained in Trinidadian-heavy oils under mining, transportation, and processing conditions, and to design modifiers and optimal operational conditions which will mitigate the nuisance they create. Atomistic MD represents bonded atoms in molecules by balls held together by springs and uses Newtonian mechanics to calculate molecular trajectories under Potential Fields using software such as GROMACS

The objective of this work is to validate the use of GROMACS to calculate the trajectories of molecules and predict: transport, thermodynamic properties and interactions leading to formation of clusters of asphaltenes. The Optimized Potential for Liquid Simulation All Atoms (OPLS_AA) (eqn. (1)), with appropriately chosen parameters is used to model hydrocarbon liquids like Trinidad's heavy oils. The symbols have their usual meaning.

It comprises potentials due to: covalent bonds, LennardJones and electrostatic forces due to polar groups. The Boltzmann Distribution of energies, the equipartition theorem with Maxwell's distribution of initial velocities of the molecules, and the periodic boundary condition (PBC) apply.

The major drawback in the present simulation techniques is that knowledge of the structure of asphaltenes remains imprecise and the molecular composition of asphaltenic oils in specific process environment is unknown. Techniques such as Quantitative Molecular Representation (QMR) ${ }^{3,4}$ are used to match possible representative structure with analytical results. Techniques including ${ }^{1} \mathrm{H}$ and ${ }^{13} \mathrm{C}$ NMR; Small Angle Neutron Scattering, X-Ray diffraction and experimental observations on reactivity products, solubility and precipitation, Small Angle X-Ray Scattering, Gel Permeation Chromatography ${ }^{4}$. For simplicity, in this validation exercise we have used the continental model asphaltene shown as $\mathrm{C}$ in figure 1 to carry out simulation using GROMACS. 
Proc. of the Seventh Intl. Conf. on Advances in Bio-Informatics, Bio-Technology and Environmental Engineering - ABBE 2018 Copyright (C) Institute of Research Engineers and Doctors, USA .All rights reserved.

ISBN: 978-1-63248-161-0 DOI: 10.15224/978-1-63248-161-0-38

Fig. 1: Types of Asphaltene Molecules 1

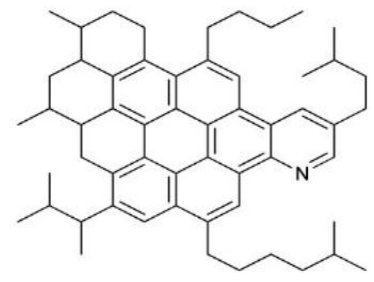

A "Continental" Asphaltene

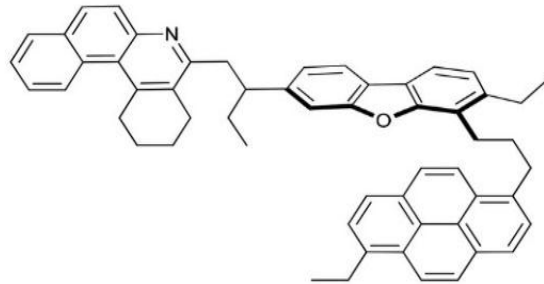

B "Archipelago" Asphaltene

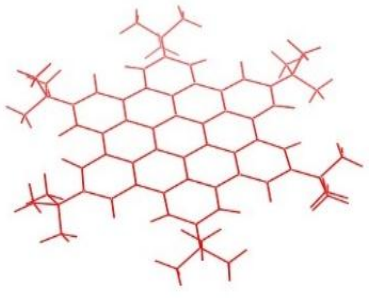

C model "Continental" Asphaltene

Equation. 1

$$
\begin{gathered}
E_{i, j}=\sum_{\text {bonds }} k_{i}^{\text {bond }}\left(r_{i}-r_{0}\right)^{2}+\sum_{\text {angles }} k_{i}^{\text {angle }}\left(\theta_{i}-\theta_{0}\right)^{2}+\sum_{\text {dihedral }} k_{i}^{\text {dihedral }}\left[1+\cos \left(n_{i} \emptyset_{i}+\delta_{i}\right)\right] \\
+\sum \sum\left\{\frac{q_{i} q_{j} e^{2}}{\epsilon r_{i, j}}+4 \epsilon_{i, j}\left[\left(\frac{\sigma_{i, j}}{r_{i, j}}\right)^{12}-\left(\frac{\sigma_{i, j}}{r_{i, j}}\right)^{6}\right]\right\}--(1)
\end{gathered}
$$

\section{The System Simulated}

A cube of size $7 \mathrm{~nm}$ was filled with 12 molecules of model asphaltene (see $\mathrm{C}$ in Figure 1) and remainder heptane to make 7wt/wt.\% model solution.

Festus Addo-Yobo ${ }^{1}$

The University of Trinidad and Tobago

Trinidad and Tobago

Adrian Lutchman ${ }^{2}$

The University of Trinidad and Tobago

Trinidad and Tobago

\section{The Method}

\section{A. Simulation steps}

Insert asphaltene molecules in simulation box and add solvent to create a configured system. Check if there is net a charge and add ions to neutralize. Minimize Energy of the initial configuration using steepest descent method. Equilibrate to the desired temperature and pressure by running the NVT ensemble (constant number of molecules, volume, and temperature) for $100 \mathrm{ps}$. Then run simulation at constant number of molecules, temperature and pressure (NPT ensemble) for 100ps. 


\section{B. Production Molecular Dynamics}

A simulation time step of 2.0 fs was used; MD simulation lasted 40ns and 100ns; Particle Mesh Ewald was used to calculate the long range electrostatic interaction; Cut off for non-bonded interaction was fixed at $1.0 \mathrm{~nm}$; Berendsen thermostat and the Parrilello-Rahman barostat setting were used with time step of 2fs. Radial Distribution Function (RDF) was used to analyze cluster formation.

\section{The Results And Discussions}

The diffusion coefficient of asphaltene in heptane was estimated from the trajectories of the molecules using Einstein's plot in Figure 2, which agrees with measured values. The transverse-current autocorrelation-function (TCAF) routine in GROMACS6.7 was used to estimate time constants in the decay of the transverse-current autocorrelation functions of the fluctuations in velocity gradients in the liquid and the viscosity 6 inferred from them. In Fig. 3 the Viscosity is plotted against $\mathrm{k}$, a function of the decay constant converges to experimental values.

Molecules separated by $3.5 \AA$ or less are considered associated and thus are part of a cluster. The events recorded after 5 ns simulation in vacuum, showed all molecules are clustered. In Figure 4, the molecules are stack laterally indicating interaction of $\pi$ electrons above and below the plane of the molecules. This is what one would expect.

Figure 5 shows the simulation box containing dispersed asphaltene and heptane molecules at the beginning of simulation. After 10 ns production of NVT simulation (Fig. 6): a few molecules are un-clustered and others are clustered in pairs and in trimers. Some molecules are attached horizontally but displaced indicating $\pi$ bonding between the aromatic cores; others are attached edgewise to the aromatic core. Edgewise attachments is not observed in MD runs in vacuo but may have been made possible due to solvation by heptane. The arrangements of the molecules in the clusters are expected to have implications for cluster growth, agglomeration, flocculation and other phase transitions.

Figure 7 shows the probability of an asphaltene molecule belonging to clusters of varying sizes including the monomer after the simulation time of 100ns. The maximum cluster size contained 5 molecules. The proportion of molecules belonging to a cluster reduced from $36 \%$ for the monomer to $12.5 \%$ for the cluster with 5 molecules

\section{v. Summary And Conclusion}

The use of GROMACS for atomistic MD simulation of asphaltene in vacuo and in heptane is validated. The diffusion coefficient of the monomer, the bulk viscosity and the density can be evaluated from the trajectories to yield realistic values in agreement with published experimental data. In vacuo, rapid aggregation at low temperatures is observed. The phenomena of molecular aggregation and clustering in heptane is observed. Two types of stacking are observed - flat stacking and edgewise association with a cluster. In heptane only a minority of molecules remain unassociated.

\section{References}

[1] P.M. Spiecker, KL Gawrya, PK Kilpatrick, J. Colloid \& Interface Sci. 267, (2003) 178-193

[2] T.F. Headen, E.S Boek, G. Jackson, T.S. Totton. E.A.Miller, Energy and Fuels, 2017, 31(2) pp 1108-1125

[3] Kowalewski, I.; Vandenbroucke, M.; Huc, A.Y.; Taylor, M.J. ; Faulon J. L. Energy Fuels 1996, 10, 97-107.

[4] Sheremata, J. M.; Gray, M. R.; Dettman, H. D.; McCaffrey, W. C. Energy Fuels, 2004, 18, 1377-1384

[5] Bruce J. Palmer. Phys. Rev. E 49, 359

[6] R. Kubo (1966), Report Progr. Phys 29, 255-259

[7] Gromacs User Manual version 5.0.4, www.gromacs.org 
Proc. of the Seventh Intl. Conf. on Advances in Bio-Informatics, Bio-Technology and Environmental Engineering - ABBE 2018 Copyright (C) Institute of Research Engineers and Doctors, USA .All rights reserved.

ISBN: 978-1-63248-161-0 DOI: 10.15224/978-1-63248-161-0-38

\section{vi. Appendix}

Table 1:

\begin{tabular}{|l|l|}
\hline$E_{i, j}$ & Interaction Potential between 2 atoms \\
\hline$k_{i}^{\text {bond }}$ & Bond stretching constant \\
\hline$r_{i}$ & Radial position \\
\hline$r_{0}$ & Radial position at equilibrium \\
\hline$k_{i}^{\text {angle }}$ & Force constant for angular displacement \\
\hline$\theta_{i}$ & Angular displacement \\
\hline$\theta_{0}$ & Equilibrium angular position \\
\hline$k_{i}^{\text {dihedral }}$ & dihedral force constant \\
\hline$\emptyset_{i}$ & Dihedral torsion angle displacement \\
\hline$q_{i}$ & Charge on molecule i \\
\hline$q_{j}$ & Charge on molecule j \\
\hline $\mathrm{e}$ & Columbic charge of electron \\
\hline$\epsilon$ & Dielectric constant \\
\hline$\epsilon_{i, j}$ & Potential energy minimum \\
\hline$\sigma_{i, j}$ & Lennard Jones Potential parameter \\
\hline$r_{i, j}$ & Distance between molecules \\
\hline
\end{tabular}


Proc. of the Seventh Intl. Conf. on Advances in Bio-Informatics, Bio-Technology and Environmental Engineering - ABBE 2018 Copyright (C) Institute of Research Engineers and Doctors, USA .All rights reserved.

ISBN: 978-1-63248-161-0 DOI: 10.15224/978-1-63248-161-0-38

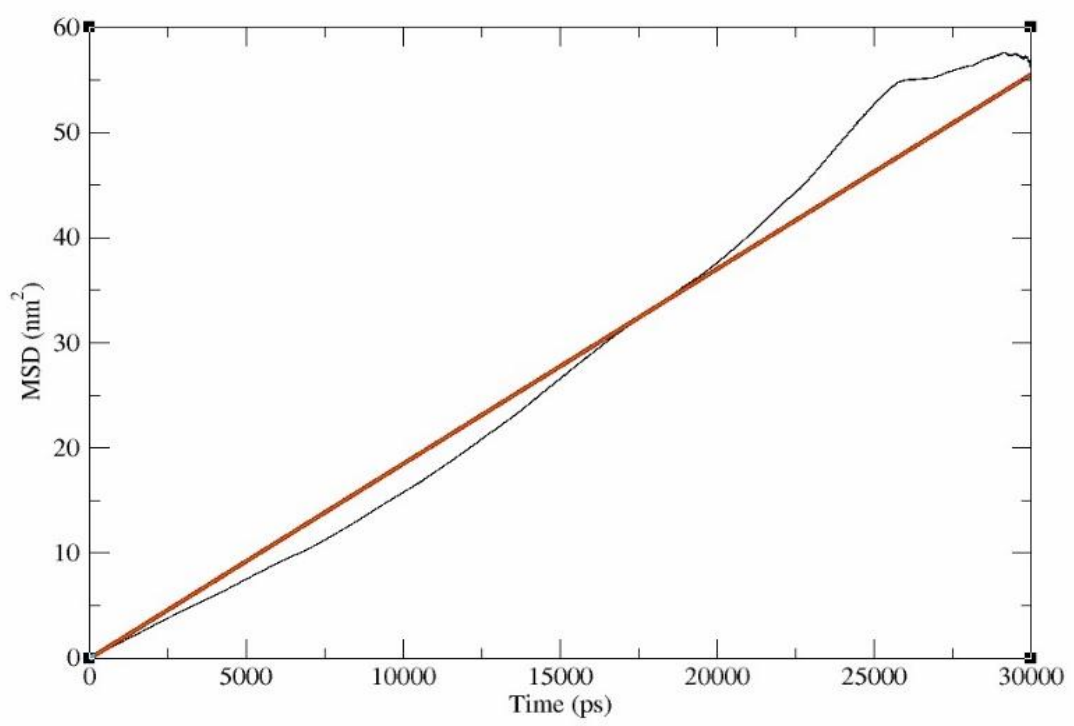

Fig. 2: mean square displacement $\langle\Delta \mathbf{r} 2\rangle$ vs Time

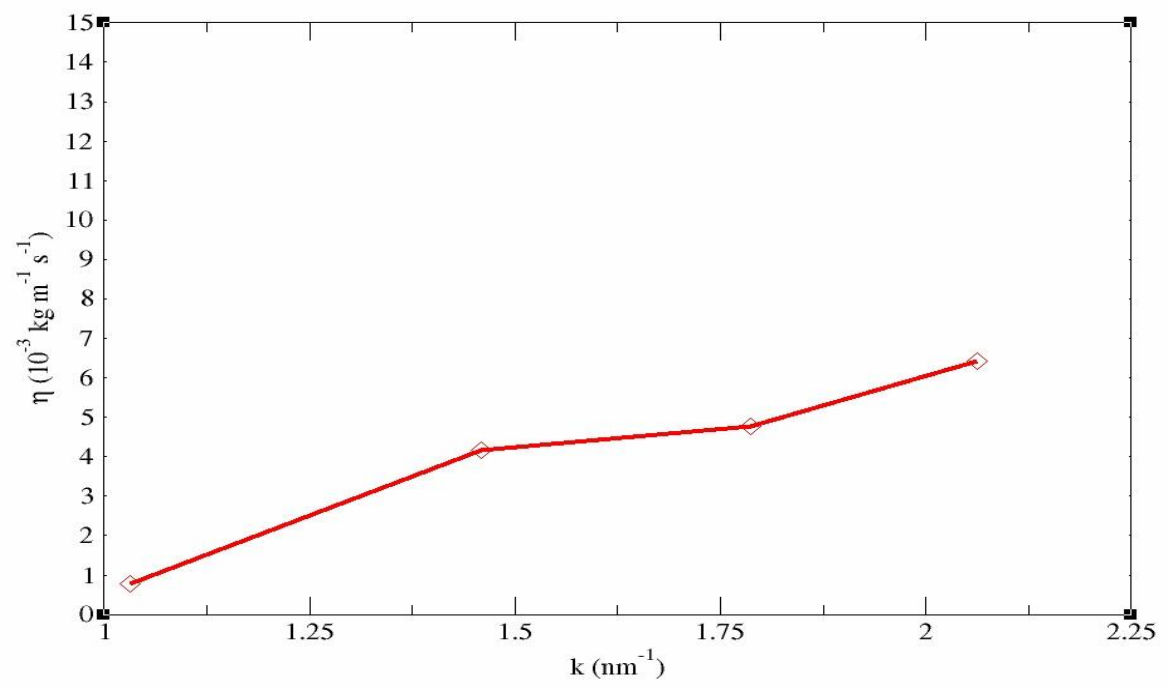

Fig. 3: Viscosity correlation 
Proc. of the Seventh Intl. Conf. on Advances in Bio-Informatics, Bio-Technology and Environmental Engineering - ABBE 2018 Copyright (C) Institute of Research Engineers and Doctors, USA .All rights reserved.

ISBN: 978-1-63248-161-0 DOI: 10.15224/978-1-63248-161-0-38

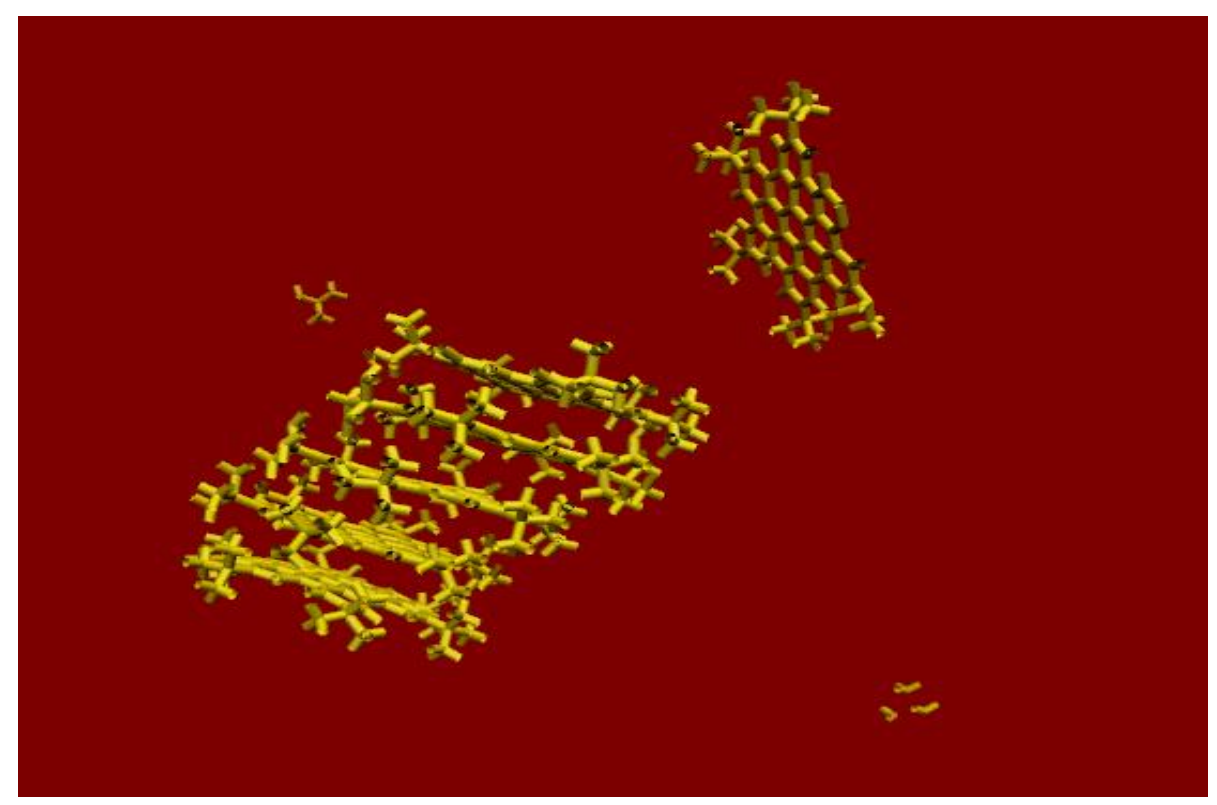

Fig. 4: Asphaltene clusters in vacuum

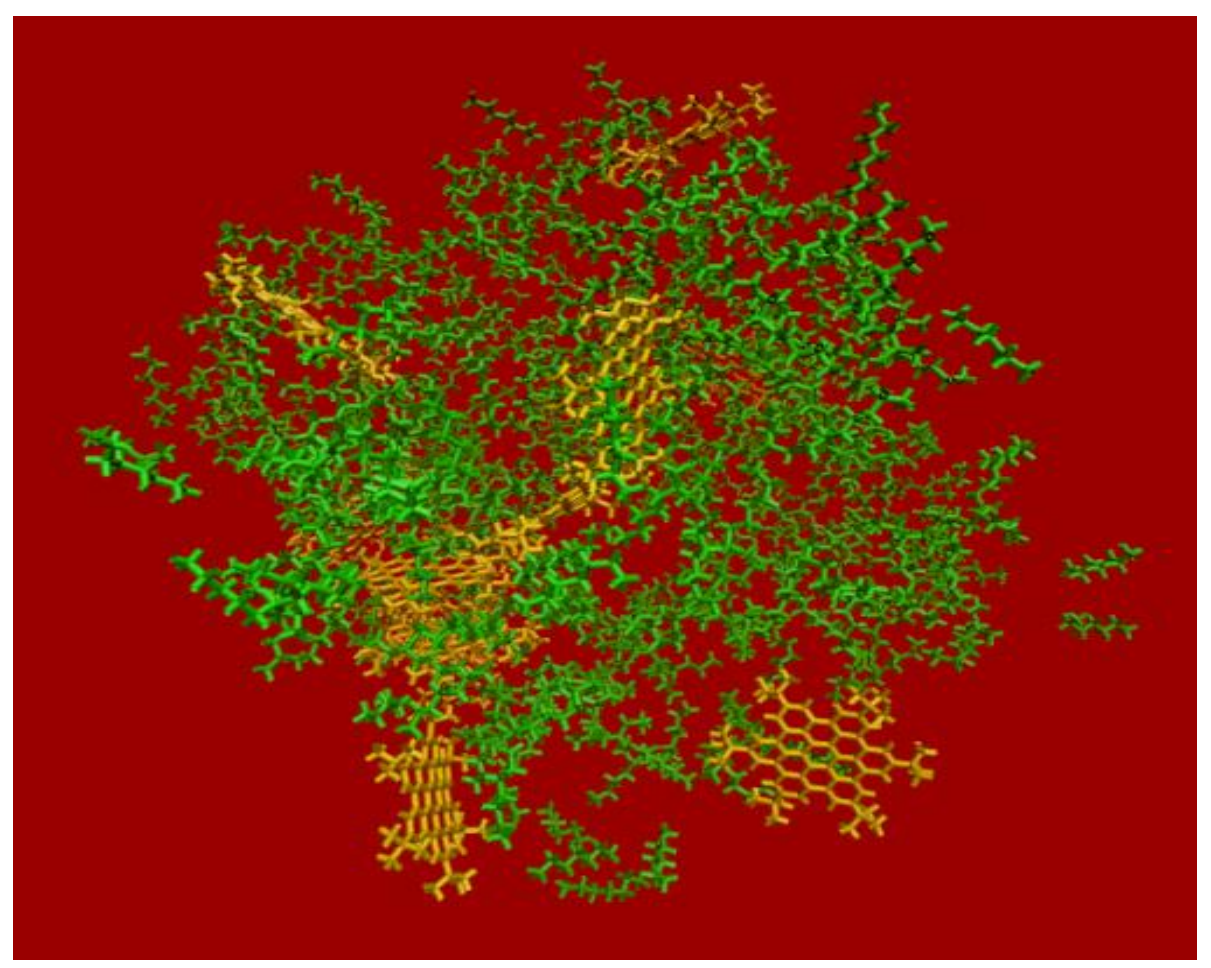

Fig. 5: Asphaltene in Heptane 


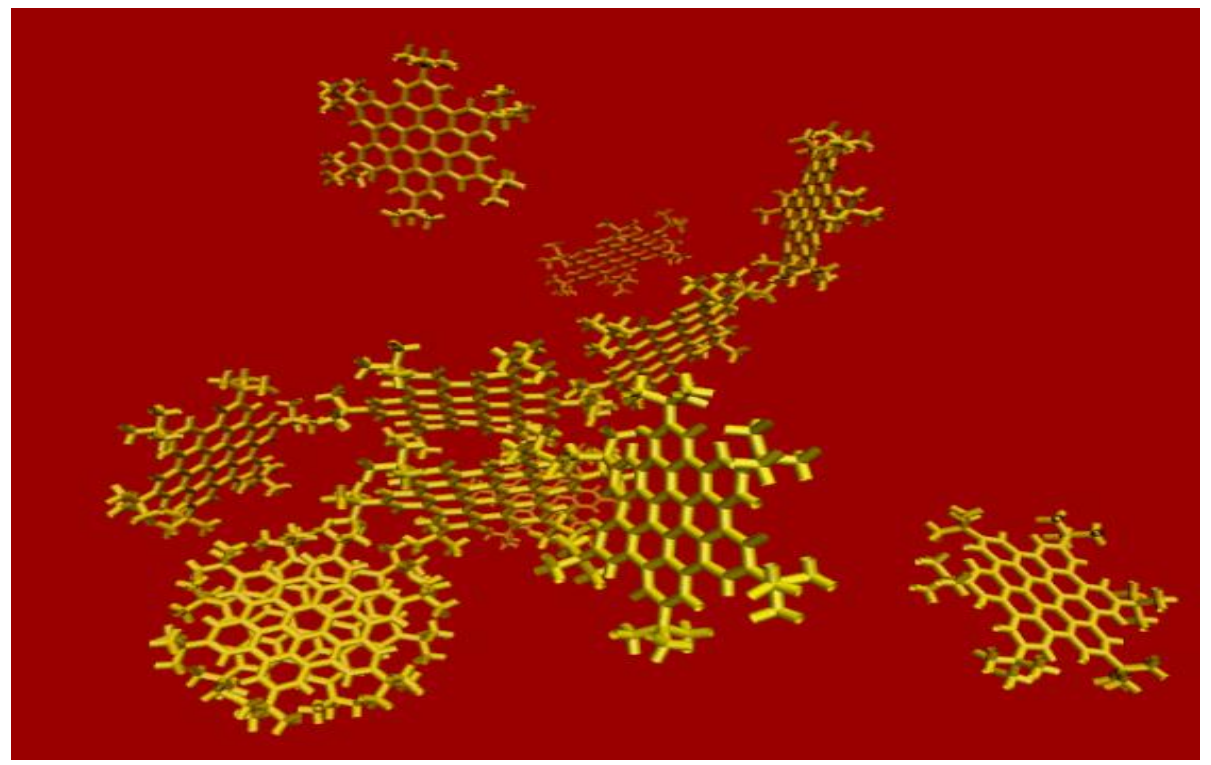

Fig. 6: Clustered and un-clustered Asphaltene

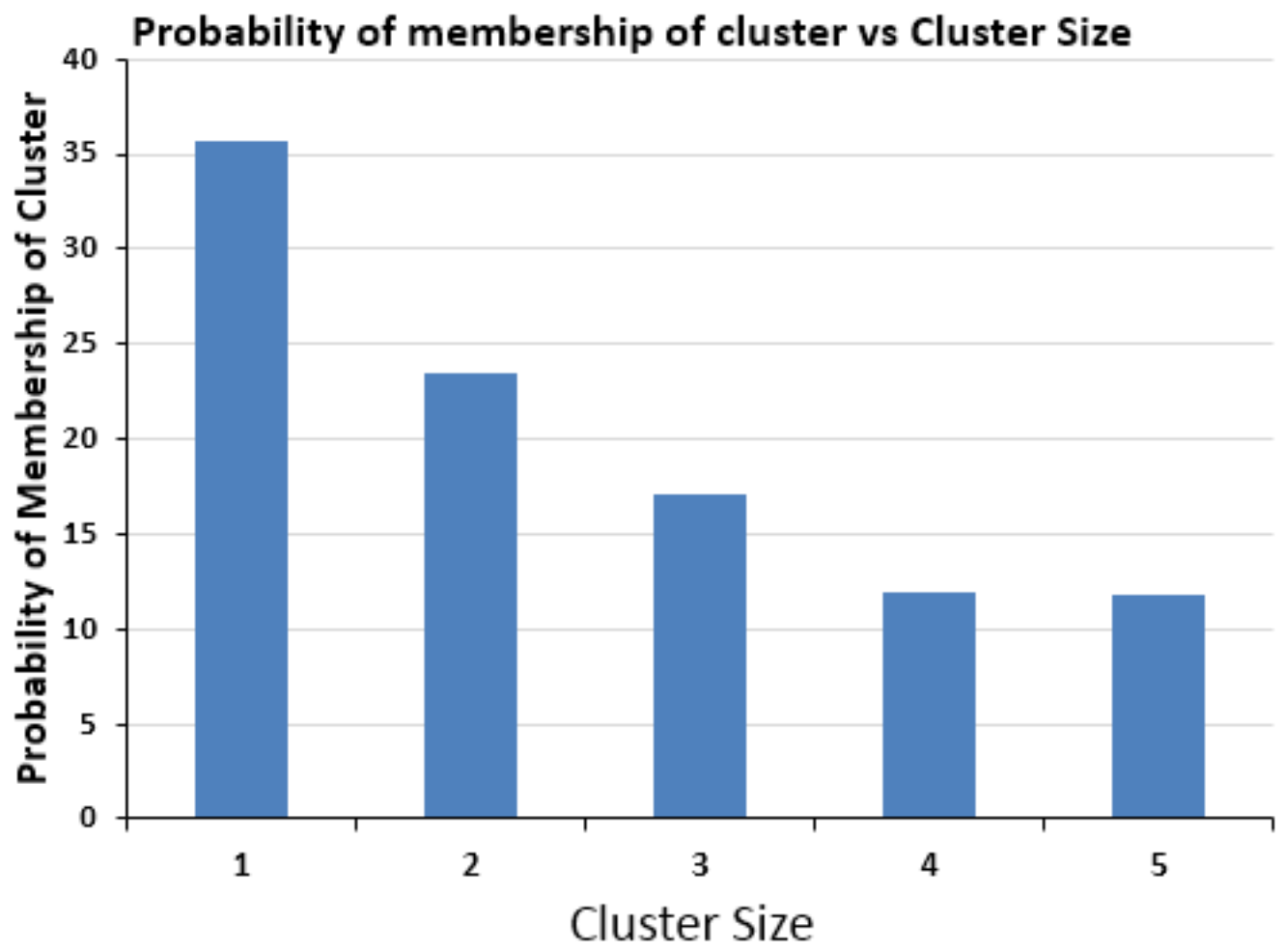

Fig. 8: Histogram of Cluster Size distribution 